\title{
3D DOCUMENTATION OF ARCHEOLOGICAL REMAINS IN THE UNDERWATER PARK OF BAIAE
}

\author{
F. Bruno ${ }^{1}$, A. Lagudi $^{1}$, A. Gallo ${ }^{1}$, M. Muzzupappa ${ }^{1}$, B. Davidde Petriaggi ${ }^{2}$, S. Passaro ${ }^{3}$ \\ ${ }^{1}$ Dept. of Mechanical, Energetics and Management Engineering, Università della Calabria, Rende (CS), Italy - \\ (fabio.bruno, antonio.lagudi, alessandro.gallo,maurizio.muzzupappa)@unical.it \\ ${ }^{2}$ Istituto Superiore per la Conservazione ed il Restauro, Nucleo per gli Interventi di Archeologia Subacquea, Rome, Italy - \\ barbara.davidde@beniculturali.it \\ ${ }^{3}$ Consiglio Nazionale delle Ricerche, Istituto per l’Ambiente Marino e Costiero, Naples, Italy - salvatore.passaro@iamc.cnr.it
}

\section{Commission V}

KEY WORDS: 3D documentation, Baiae Archeological Park, 3D reconstruction, 3D model accuracy, optical and acoustic data merging

\begin{abstract}
:
This paper presents some experimentations, which have been conducted in the submerged archeological Park of Baiae, aimed to identify the problems related to the underwater 3D documentation process. The first test has been addressed to verify if a dense stereo mapping technique, usually employed in terrestrial and aerial applications, might be employed in critical underwater conditions by assessing the influence of different factors on the results. In the second test, the accuracy of the 3D model obtained through this technique has been evaluated. The third test deals with the geo-localization of the 3D models, conducted by merging the optical and acoustic data, through a multi-resolution bathymetric map of the site as a reference.
\end{abstract}

\section{INTRODUCTION}

3D reconstruction offers many opportunities for the documentation of underwater cultural heritage, thanks to the possibility to de-materialize the tangible heritage, so that it can be enjoyed and studied without any constraint given by the marine environment. 3D data of underwater scenes are usually acquired through optical or acoustic sensing technologies. Acoustic systems typically give good results at long distances and in presence of bad visibility, but the resulting 3D data are affected by low resolution and accuracy, and do not contain color information. Optical techniques give better performances in presence of clear water and in close range applications.

Passive optical techniques are very promising for the documentation of underwater cultural heritage, as they offer the required resolution, and can be obtained with an off-the-shelf camera handled directly by a scuba diver.

On the other side, optical imaging suffers from the harsh conditions caused by several environmental factors, such as light absorption and color alteration induced by the water, turbidity caused by the particles suspended in water, presence of moving elements like algae or fishes. These problems limit the use of optical techniques to close range applications, and require a proper image pre-processing to improve the quality of the $3 \mathrm{D}$ model.

In this paper we discuss various issues concerning the 3D reconstruction of underwater archeological sites though the use of photogrammetric techniques. In particular, we analyze the performances of the dense stereo mapping technique commonly used in air - highlighting its limits in underwater environment.

Another research line focuses on the integration of 3D data captured by acoustic and optical systems, in order to obtain a complete representation of the underwater scene and to geolocalize the optical 3D model using the bathymetric map as a reference. Since the two categories of sensors are based on different physical principles, data alignment, (i.e. their transformation from each sensor's local frame into a common reference frame) is of critical importance for the successful integration of the optical and acoustic 3D models. Indeed, the $3 \mathrm{D}$ data generated by these two sensors are very different in terms of resolution and accuracy, so that the alignment made through the recognition of common points in both representations is a complex task.

In the following sections we present three case studies, describing the critical aspects encountered during the 3D documentation process in the context of the submerged archeological Park of Baiae (Naples).

\section{ARCHEOLOGICAL CONTEXT: THE SUBMERGED ARCHAELOGICAL PARK OF BAIAE}

The underwater archaeological site of Baiae is located a few kilometers North of Naples (Italy) and belongs to the majestic volcanic complex of Campi Flegrei (Figure 1).

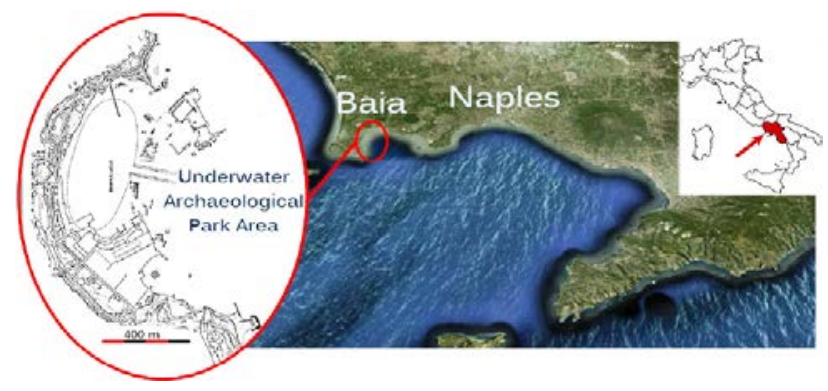

Figure 1. Geographical localization of underwater archeological site of Baiae (Italy).

Today Baiae is a small village in the municipality of Bacoli. Despite its limited size, it is dense of ancient remains of exceptional archaeological, historical, and architectural importance.

The archaeological submerged area of Campi Flegrei extends seamlessly along the entire coastline from Cuma to Naples. The depth of the sites ranges from $0.0 \mathrm{~m}$ to $15.00 \mathrm{~m}$ ca.

In 1997, the archaeological submerged area of Baiae became a restricted area. In 2002, the Ministry of Culture and the Ministry 
of Environment have established the Marine Protected Area of Baiae - Underwater Archaeological Park (AMP Baiae).

The area was divided into three zones with different levels of protection, according to the density of submerged archaeological remains.

The archaeological submerged city of Baiae stands as a direct connection with the past: its extension (176,600 hectares), historical importance, and inestimable value make it an ideal place for studying and understanding the ancient world.

The underwater archaeological park includes a number of architectural remains of villae maritimae. A number of walkways, gardens (viridaria), thermae, landing ports, fish ponds, plunge pools, floors (opus tessellatum, opus sectile, opus signinum, opus spicatum), decorated plasters, and artistic artifacts as statues, pottery, glassware and polychrome mosaics embellish and adorn the submerged structures. Wooden relics of building structures still show those ancient construction techniques that reached their utmost expression in Baiae (Baiae's pozzolana was the most famous of the ancient world and was used all around the Mediterranean, as the ships loaded with pozzolana used to sail from Portus Iulius to East).

The site is recognizable from the ancient ruins located on the banks and the access channel at about $-6 \backslash 8 \mathrm{~m}$., in the centre of the roadstead, cutting the Isthmus that separated it from the sea. To the east of the nymphaeum of Claudius (Ist c. A.D., exc. 1981/82) and the stone-paved road toward the foothills (Ist-IVth c. A.D., exc. and rest. 2008), there are some thermae (Ist-IIIrd c. A.D.) and a villa dating back to the age of Hadrian (117-138 AD) located south-east of Punta Epitaffio, built on the remains of an older villa (late Ist B.C.-early Ist c. A.D.), attributed to the Pisones family.

The vast complex featured thermae, gardens, and a maritime quarter, with residential rooms, cisterns, fish ponds and two landing sites, protected by breakwaters, partly similar to Villa Adriana (Tivoli), and it has probably been built under the direct intervention of Hadrian. Other remains have been found beyond the channel and on the seabed in front of the Castle of Baiae.

As it is well known, the ancient submerged remains are subjected to erosion phenomena, manmade damage, and colonization by biological agents. All these factors are problematic for restoration and preservation, and involve high maintenance costs, as reported during the ISCR experimental restorations (2003-2014) and the P.O.R. of Baia Sommersa (Caputo and Severino, 2008); the restored pathways are currently in need of maintenance.

In the past years the ISCR, with the project 'Restoring Underwater' (Petraggi, 2004; Petraggi et al., 2012; Petraggi et al., 2013), has restored some sectors of ancient villas and structures situated in the Park of Baiae: the Villa con ingresso a Protiro, the Villa dei Pisoni, the Via Herculanea and the Edificio con cortile porticato at Portus Iulius.

This case study is particularly critical because of the high level of turbidity, the heavy presence of marine flora and the variable lighting conditions, commonly found in shallow waters.

All these disturbances cause a decreasing of the contrast and an alteration of the colors in the acquired images, seriously affecting the 3D reconstruction. So we were not sure about the feasibility of using a multi-view photogrammetric technique for obtaining a sufficiently detailed model of the architectonic structures present in the site.

\section{3D RECONSTRUCTION IN UNDERWATER ENVIRONMENT}

For the 3D reconstruction of submerged architectonic structures and objects, underwater archaeology researchers can employ both acoustic and optical technologies.

Multibeam echosounders (MBES), usually employed for the generation of multi-resolution bathymetric maps in archeological contexts, give good results at long distances and in presence of bad visibility, but the resulting 3D data are affected by low resolution and accuracy, and do not contain color information. Optical techniques give better performances in presence of clear water and in close range applications.

Among the optical techniques, photogrammetry has known a notable development during the last decade (Bruno et al., 2011; Silvatti et al., 2012), with a particular attention for the dense stereo mapping technique (Lowe, 2004). The latter, compared to the active ones, allows for a fast and accurate 3D survey through standard photographic equipment (Bianco et al. 2013). Other approaches, based on underwater passive photogrammetry, which integrate camera calibration procedures and image correction and enhancement methods, have been effectively applied in underwater environment for the survey of archaeological sites, as in the case of Hyères (France) where the Grand Ribaud Etruscan Wreck lies in deep water (Drap et al., 2001; Drap et al. 2008), and the shipwreck of Dolia in the Tuscan Archipelago (Sorbi et al., 2014).

The 3D reconstruction process starts with the orientation of a sequence of images taken from different viewpoints, using a structure-from-motion technique. In the first stage, a feature extraction algorithm such as the SIFT (Scale Invariant Feature Transform) is used to extract a set of descriptors across the collection of images to be matched. Subsequently, a bundle adjustment procedure computes the camera positions and retrieves the camera calibration parameters.

In the second stage, a passive dense stereo matching algorithm identifies some corresponding points across the image sequence. Knowing these correspondences and the scene structure, the $3 \mathrm{D}$ world coordinates of each image point can be reconstructed by triangulation. Finding a pair of correspondent points is not so trivial; since the scene is acquired from different points of view, it is possible to find false correspondences due to occlusion, radiometric distortion, and perspective distortion.

Stereo systems use two digital synchronized cameras which may be installed on underwater robots (Allais et al., 2007). The use of three synchronized cameras has also been experimented for improving accuracy in underwater surveys conducted by a scuba diver (Menci, 2013).

\section{CASE STUDY 1: PRELIMINARY TEST ON VILLA CON INGRESSO A PROTIRO}

The first study was aimed to verify if the dense stereo mapping technique, usually employed in terrestrial and aerial applications, might be employed in the critical conditions of the Baiae site, and to assess the influence of different image enhancement algorithms on the 3D reconstruction results.

As mentioned in the previous sections, the archaeological site of Baiae presents several peculiarities compared to other underwater sites. In fact, we know that the Baiae environment conditions, in terms of water turbidity and presence of flora, are very critical and we were not sure about the possibility of using a multi-view photogrammetric technique for obtaining a sufficiently detailed model of the structures present within the site.

The area selected as case-study is a thermal room, a caldarium of Villa con ingresso a Protiro, sized 5 × 8 meters. The caldarium is delimited by a wall with an elevation ranging around 0.5 meters. There is one crack on the west side and it presents several blocks of bricks spread over the floor.

This particular room has been selected because, it presented various materials (bricks, mortar, tile floors, etc.) at the time of the acquisition, and it was affected by a strong colonization of algae and other kind of bio-fouling, thus constituting a very hard test case for the 3D acquisition techniques.

There are several methods in the state of the art that can be used to improve the 3D reconstruction quality through the 
enhancement of underwater images, and a number of parameters have a relevant impact on the results. In order to identify how these parameters affect the accuracy of the reconstructed model, we have performed a rigorous analysis of the factors involved in the 3D reconstruction process.

\subsection{Image acquisition}

The camera used to acquire the underwater pictures is a Nikon D7000 reflex camera with a $20 \mathrm{~mm}$ fixed lens. The camera is mounted in an underwater housing manufactured by Ikelite, equipped with a spherical port. In addition, two underwater strobes have been used to acquire pictures in some portions of the site that present very poor lighting conditions.

The acquisition has been carried out in two different dive sessions, in the North part and the South part of the site, respectively.

The reflex camera has been used in manual exposure mode, setting values between $1 / 250$ and $1 / 320$ of a second for the exposure time and an aperture between $\mathrm{f}-5.6$ and $\mathrm{f}-8$. In order to ensure the adaptation of exposure to variable lighting conditions and to avoid the acquisition of over-exposed and under-exposed areas, the automatic ISO sensitivity function has been used. In order to test the effects of the in situ white balance correction, the images have been acquired using the pre-measured value obtained by acquiring an underwater white balance panel by Lastolite. The pictures have been taken following a standard aerial photography layout: the diver swims at depth of about 2 meters, taking overlapping pictures along straight lines that cover the whole area in North-South direction. Another set of images has been acquired in East-West direction. The occluded areas, not visible in vertical pictures, have been acquired using oblique photographs. The complete dataset includes a total of about 722 images.

In this experimentation, the original images have been processed through an histogram stretching and a manual color retouching procedure performed on a sample image of the dataset, and it has been implemented using batch actions in Adobe Photoshop ${ }^{\circledR}$ to rescue the maximum amount of information from a set of defective and noisy pictures.

\subsection{Experimentation and results}

For the reconstruction process, the Bundler software (Snavely et al., 2008) has been used to orient the images and to retrieve the camera calibration parameters.

The use of enhanced images allowed for orienting a subset of 533 pictures covering the whole area. Subsequently, the Bundler's outputs and the undistorted images have been processed with the PMVS2 (Patch-based Multi-View Stereo) algorithm (Furukawa and Ponce, 2010). This algorithm estimates the surface orientation while strengthening the local photometric consistency, which is important to obtain accurate models from low textured objects or with images affected by blur due to turbidity in underwater environment. Furthermore, the PMVS2 automatically rejects moving objects, such as fishes and algae.

The output is a dense point cloud (about 10 million points) with RGB information for each 3D point (Figure 1).

The point cloud has been processed with Meshlab tools (Meshlab, 2014). The first operation was a manual selection and deletion of unwanted areas. Subsequently, a water tight surface with about 25 million triangles has been obtained through a Poisson Surface Reconstruction algorithm.

The camera orientation procedure has been carried out with an unknown scale factor and it was followed by a scaling of the model, which was carried out by selecting two points with a known distance. In our experimentation, a scale bar has been placed in the scene and reconstructed in order to evaluate the scale factor.

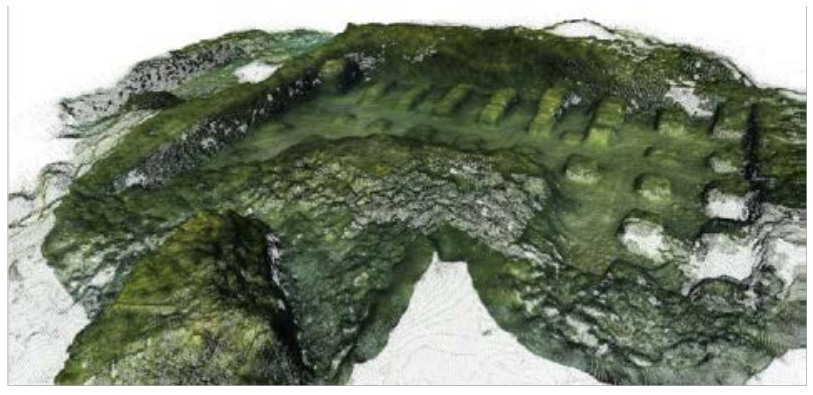

Figure 1. Reconstructed dense point cloud

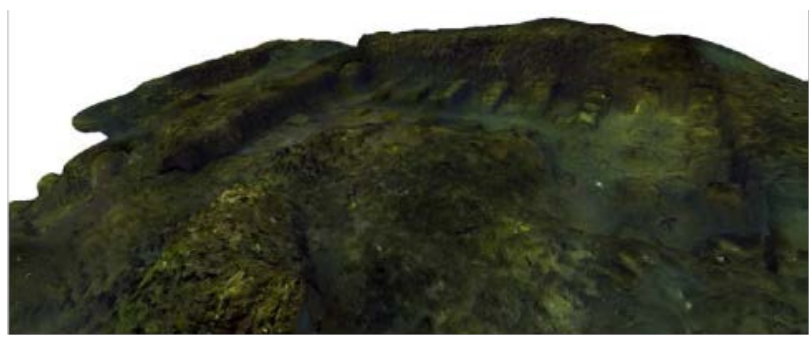

Figure 2. Final textured 3D model

The last step consists in the application of the texture on the 3D surface. Color information can be extracted directly from the colored point cloud, but this method does not allow for creating a high quality texture, as its resolution depends on the point cloud density.

Since the camera positions are known, the method chosen for texture mapping consists into the projection and blending of the high resolution images directly on the 3D surface. In particular, we have selected an image subset because the averaging among neighborhood values during the blending on the images works better if performed on a largely overlapped area (reducing blur effects). The result of this procedure is a texture with a resolution comparable to the original images (Figure 2).

\section{CASE STUDY 2: ACCURACY EVALUATION} ON VILLA DEI PISONI

In this second case study, we have evaluated the accuracy of the reconstruction method described in Section 4. This experimentation has been carried out in the "Villa dei Pisoni".

Since a reference model acquired with an active sensor was not available, the data have been analyzed - in terms of distance measurements accuracy - on rigid targets placed on the seabed. The aim of the work is to quantify the accuracy of the 3D model of an underwater structure generated by an image-based method, in order to verify its applicability on challenging real word scenarios.

Villa dei Pisoni is located at about $150 \mathrm{~m}$ southeastward from the Punta Epitaffio Cape and is also visible from aerial and satellite images. It is composed by several rooms with a global rectangular plan structure measuring $160 \times 120 \mathrm{~m}$. Most of the internal sector was occupied by gardens (about $95 \times 65 \mathrm{~m}$ ) and surrounded by apses and columns. A fistula aquaria containing the inscription "L. Pisonis" confirms that the Villa belonged to the important "Pisonis" family and was dating back to the first century BC (Hadrian age). 


\subsection{Experimental setup and 3D reconstruction}

The equipment used to acquire the underwater pictures consists in a Nikon D7000 reflex camera with a $20 \mathrm{~mm}$ fixed lens. The camera is mounted in an underwater case manufactured by Ikelite and equipped with a spherical housing.

In order to compute the global scale, a set of four triangular targets, made of rigid bars of anodized aluminium, each with three markers placed at the vertices of the triangle, has been used to perform

three measurements, corresponding to the distances between each pair of markers.

Moreover, a four meters long rigid bar, also made of anodized aluminium, has been used to analyze the accuracy (Figure 3).

The acquisition was separated into three

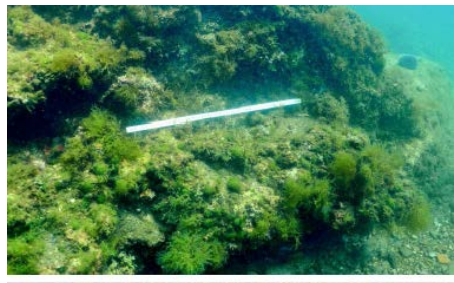
sessions carried out in three days. During the first dive, a preliminary survey of the site has been conducted, in order to study the area of interest and identify the most relevant architectonic structures, monitor the environmental conditions, and find the best places on which the targets should be placed. Due to the extension of the area of interest ( 15 x 20 meters), we have decided to divide the acquisition into two different sessions. The first dive, which lasted about two hours, was conducted in the northern part ( $15 \times 10 \mathrm{~m})$ : about 1700 pictures have been acquired. During the afternoon of the same day, the picture coverage has been checked in order to avoid missed areas. The day after, more than 2000 pictures related to the southern part (15 x $12 \mathrm{~m})$ have been acquired, paying attention to ensure a sufficient overlap between the two parts to consolidate the model alignment.

The pictures have been taken following a standard aerial photography layout: the diver swims above the site at a fixed depth ranging from 1.5 to 3 meters, taking pictures with a $75 \%$ of overlapping between two consecutive photos. The camera follows two straight lines in North-South and East-West directions; several overlapping stripes have been acquired covering the whole area.

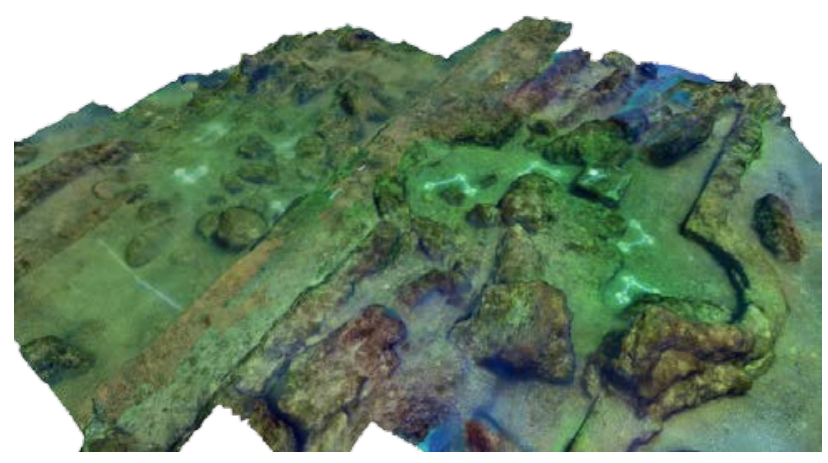

Figure 4. Final textured 3D model of Villa dei Pisoni

Vertical walls and features which are occluded in aerial pictures, have been acquired using a series of oblique photographs taken swimming around each feature.
Using the methods from our first case study we've obtained the 3D model as pictured in Figure 4.

\subsection{D model accuracy evaluation}

To recover the unknown scale factor, it was necessary to obtain accurate measurements of the scene. We measured multiple distances, and the resulting scale factor has been evaluated as the mean value of the ratios between the measurements on the real scenario and the reconstructed one. By applying this scale factor to the model, we obtained a mean error of $2.89 \mathrm{~mm}$.

Since there is no reference 3D model obtained with active techniques, it is not possible to make an accurate comparison for testing the data accuracy. That's why we used the four meters long, rigid anodized aluminum bar, on which we attached a series of markers placed at fixed distances.

After having identified these markers in the reconstructed model, we evaluated their relative distance by taking 37 measurements. We've evaluated an average error of $1.35 \mathrm{~mm}$, which is comparable to the mean error of the scaling procedure that we previously obtained.

As an additional parameter for the assessment of the accuracy of the reconstruction, we measured the geometric tolerance of straightness, which sets the limits of variability between a real straight element and the ideal one.

In our case, the straightness is defined as the minimum diameter of the cylinder that contains the whole series of markers.

We've measured a mean radius of $1.42 \mathrm{~mm}$, evaluated as the average distance between the markers and the straight line of best fit of the set of measurements.

\section{CASE STUDY 3: OPTO-ACOUSTIC DATA MERGING}

The third case study concerns the merging of optical and acoustic data in order to improve the understanding of the acquired scene (by contextualizing it in a larger bathymetric model) and to geo-localize the 3D optical models using the bathymetric map as a reference.

In the first step, two areas of the archeological site of Baiae, i.e. the fishpond area near the Castle and the Villa dei Pisoni, were acquired by using our own optical equipment and a multi-beam sonar.

Multibeam echosounders (MBES) are standard instruments used to simultaneously collect several depth measurements called "beams", and are particularly suitable for high-resolution mapping in very shallow water (up to $80 \mathrm{~m}$ depth). This equipment is particularly reliable for data acquisition in areas of archaeological interest (Passaro et al., 2013: Somma et al., 2015). The bathymetric acquisition system was properly interfaced with a differential global positioning system, which ensured a sub-meter accuracy, and with inertial motion sensors for pitch, roll, yaw and heading corrections. MBES data processing was carried out with Reson-Thales PDS2000@ software, following IHO standards (IHO, 2008). The processing was mainly aimed at the removal of navigational errors, noise reduction (de-spiking, removal of poor quality beams), and tidal and sound velocity corrections (e.g., de Alteriis et al., 2003).

Subsequently, the optical 3D models of the acquired areas have been aligned through the manual selection of corresponding points in both representations.

While in the first test we've obtained a good alignment of the 3D optical and acoustic data (Figure 5), in the second one the result was not satisfactory, because the data-sets were too different in resolution (Figure 6).

In particular, in order to have a correct alignment of the optical and acoustic 3D models, we have estimated that the data-sets 
have to contain sharp corners with a size at least three times the resolution of the bathymetric map itself (0,1 m approx.).

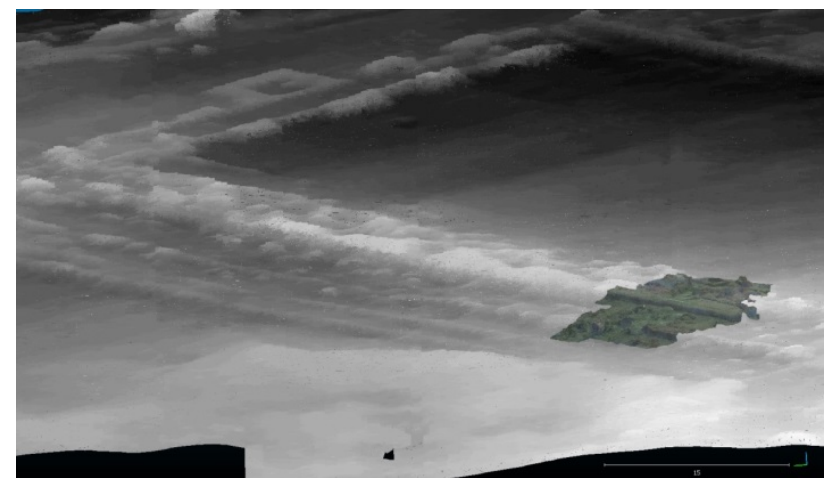

Figure 5. Opto-acoustic alignment of the Villa Pisoni.

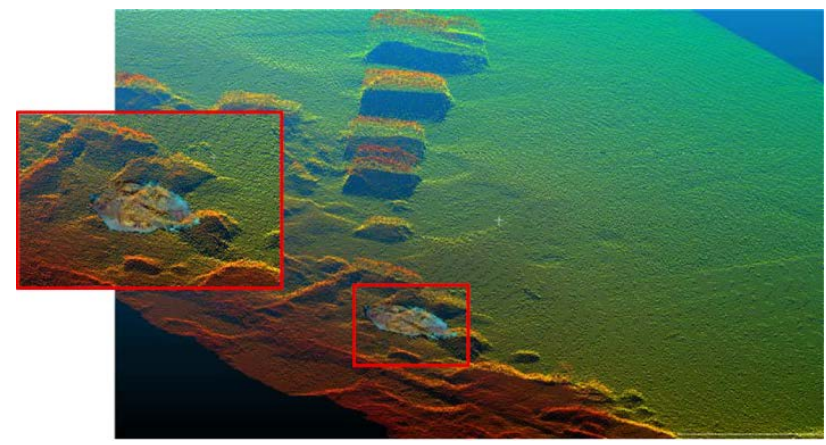

Figure 6. Opto-acoustic alignment of the fishpond area.

\section{CONCLUSION}

The paper presented three case studies about the underwater 3D documentation of an underwater archeological site, highlighting various difficulties and critical aspects.

In the first case study we have conducted a preliminary test in order to verify if the dense stereo mapping technique can be applied in a challenging underwater environment as the Baiae submerged archaeological park.

In the second case study, we have evaluated the accuracy of the 3D models obtained through the methodology adopted in the previous case study.

The accuracy of the 3D model was quantified in terms of the average error between the actual size of various targets placed on the seabed (long rigid bars made in anodized aluminium, on which we have attached a series of markers placed at a fixed distance) and the size measured in the 3D reconstruction. As an additional parameter for the assessment of the accuracy of the reconstruction, the geometric tolerance of straightness has been measured. The results showed that this models can be used not only for illustrative purposes, but also for scientific analysis.

Finally, the third case study concerns the merging of data acquired by the dense stereo mapping and by a multi-beam sonar in order to improve the understanding of the acquired scene (by contextualizing it in a larger bathymetric model) and to geo-localize the 3D optical models using the bathymetric map as a reference. In the first step, two areas of the archeological site of Baiae have been acquired using a multibeam sonar, i.e. the fishpond area near the Baiae Castle and the Villa dei Pisoni. Subsequently, the optical 3D models of the acquired areas have been aligned through the manual selection of corresponding points in both representations. While in the first test we obtained a good alignment of the 3D optical and acoustic data, in the second one the result was not satisfactory because the data-sets were too different in resolution. In particular, in order to have a correct alignment of the optical and acoustic 3D models, we have estimated that the data-sets have to contain sharp corners with a size at least three times the resolution of the bathymetric map itself.

\section{ACKNOWLEDGEMENTS}

The first and the second case studies have been supported by the "CoMAS" Project (Ref.: PON01_02140 - CUP: B11C11000600005), financed by the MIUR under the PON 'R\&C' 2007/2013 (D.D. Prot. n. 01/Ric. 18.1.2010). The third case study has been supported by the "VISAS" Project (Ref.: PAC02L2_00040 - CUP: H28C13000060005) financed by the MIUR under the PAC (D.D n. 436 - 13/03/2013).

\section{REFERENCE}

Allais, A. G., Brandou, V., Dentrecolas, S., Gilliotte, J. P., and Perrier, M. 2007. IRIS-A vision system to reconstruct natural deep-sea scenes in 3D. In The Seventeenth International Offshore and Polar Engineering Conference. International Society of Offshore and Polar Engineers

Bianco, G., Gallo, A., Bruno, F., Muzzupappa, M., 2013, A comparative analysis between active and passive techniques for underwater 3D reconstruction of close-range objects. Sensors (Switzerland), Vol. 13, N.8, pp. 11007-11031.

Bruno, F., Bianco, G., Muzzupappa, M., Barone, S., \& Razionale, A. V., 2011. Experimentation of structured light and stereo vision for underwater 3D reconstruction. ISPRS Journal of Photogrammetry and Remote Sensing, 66(4), 508-518.

Bruno, F., Gallo, A., De Filippo, F., Muzzupappa, M., Davidde, B., Caputo P., 2013, 3D documentation and monitoring of the experimental cleaning operations in the underwater archaeological site of Baia (Italy). Proceedings of the DigitalHeritage 2013 - Federating the 19th Int'l VSMM, 10th Eurographics GCH, and 2nd UNESCO Memory of the World Conferences, Plus Special Sessions fromCAA, Arqueologica 2.0. et al. Volume 1, 2013, Article number 6743719, Pages 105112

Caputo, P., Severino, N., 2008. Parco sommerso di Baia: il restauro paesaggistico-ambientale di Punta Epitaffio, il recupero della strada Erculanea e i servizi per la fruizione delle aree archeologiche sommerse. in F. Escalona, R. Ruggiero, Il progetto Integrato Campi Flegrei, Napoli, Electa, p. 56.

de Alteriis G., Passaro S. Tonielli R., 2003. New, high resolution swath bathymetry of Gorringe Bank, Eastern Atlantic”. Marine Geophysical Researches, 24, 223-244.

Drap, P., Long, L., Durand, A., and Grussenmeyer, P. 2001. From underwater photogrammetry to a web integrated documentation system: The case of the 'grand ribaud $\mathrm{F}^{\prime}$ estruscan wreck. In CIPA 2001 International Symposium, Germany, pp. 1-8.

Drap, P., Scaradozzi, D., Gambogi, P., \& Gauch, F. 2008. Underwater Photogrammetry for Archaeology-The Venus Project Framework. In GRAPP, Funchal, Madeira - Portugal, pp. 485-491. 
Furukawa, Y., Ponce, J., 2010. Accurate, Dense, and Robust Multi-View Stereopsis. Pattern Analysis and Machine Intelligence, IEEE Transactions on, 32 (8), 1362-1376.

IHO-International Hydrographic Organization 2008. IHO Standards for Hydrographic Surveys. Special Publication no. 44 Published by the International Hydrographic Bureau, Monaco, pp. 28.

Lowe, D. G., 2004. Distinctive image features from scaleinvariant keypoints. International Journal of Computer Vision, 60(2), 91-110

Menci. Webpage: http://www.menci.com. (June 2014).

MESHLAB. Webpage: http://meshlab.sourceforge.net (June 2014).

Passaro S., Barra M., Saggiomo R., Di Giacomo S., Leotta A., Uhlen H., Mazzola S., 2013. Multi-resolution morphobathymetric survey results at the Pozzuoli-Baia underwater archaeological site (Naples, Italy). Journal of Archaeological Science, 40 (2), 1268-1278.

Petriaggi, R., 2004. Restauro subacqueo delle strutture sommerse della peschiera romana di Torre Astura, "Lazio e Sabina", 2, Roma 2004, pp.273-276.

Petriaggi, R., Davidde, B., 2012. The ISCR Project 'Restoring Underwater': An Evaluation of the Results After Ten Years, Conservation and management of archaeological sites, vol. 14, 2012, pp. 192-199.

Petriaggi, R., Davidde Petriaggi, B., Ricci, S., 2013. I musei sott'acqua/Underwater Museums, in Il Restauro in Italia, arte e tecnologia nell'attività dell'Istituto Superiore per la Conservazione ed il Restauro, Roma, 2013, pp. 158-165.

Rizzi, A., Gatta, C., 2004. From Retinex to Automatic Color Equalization: issues in developing a new algorithm for unsupervised color equalization. Journal of Electronic Imaging, $13,75-84$.

Silvatti, A. P., Salve Dias, F. A., Cerveri, P., and Barros, R. M., 2012. Comparison of different camera calibration approaches for underwater applications. Journal of biomechanics, 45(6), 1112-1116.

Snavely, N., Seitz, S.M., Szeliski, R., 2008. Modeling the World from Internet Photo Collections. International Journal of Computer Vision, 80 (2), 189-210.

Somma R., Iuliano S., Matano F., Molisso F., Passaro S., Sacchi M., Troise C., De Natale G.. 2015. High resolution morphobathymetry of Pozzuoli Bay, Southern Italy. Journal of Maps, DOI: 10.1080/17445647.2014.1001800

Sorbi, L., Scaradozzi, D., Conte, G., and Gambogi, P., 2014. Geoposition data aided 3D documentation of archaeological and biological sites. In Proceedings of Meetings on Acoustics Vol. 17, No. 1, p. 70-88. Acoustical Society of America

Tonazzini, A., Salerno, E., Mochi, M., Bedini, L., 2004. Blind Source Separation Techniques for Detecting Hidden Texts and Textures in Document Images. Image Analysis And Recognition Lecture Notes in Computer Science, 3212, 241248. 